\title{
Aortic dissection in a young man with Loeys-Dietz syndrome
}

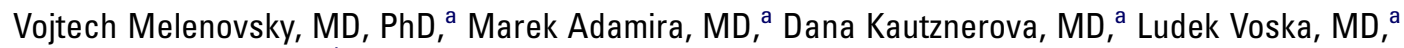 \\ Jiri Weichet, MD, PhD, ${ }^{\mathrm{b}}$ Bart Loeys, MD, PhD, ${ }^{\mathrm{c}}$ and Jan Pirk, MD, PhD, ${ }^{\mathrm{a}}$ Prague, Czech Republic, and Ghent, Belgium
}

\section{Supplemental material is available online.}

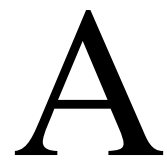

ortic dissections occur typically in older hypertensive patients with a peak incidence in the sixth decade, but occasionally they occur in younger patients, frequently with atypical presentation. Aortic dissection in this younger group is most often related to an underlying connective tissue disease, such as Marfan syndrome, vascular Ehlers-Danlos syndrome, nonsyndromic familial throacic aortic aneurysm with dissections, or the recently described Loeys-Dietz syndrome. We report the case of a young patient with Loeys-Dietz syndrome who underwent aortic dissection.

\section{Clinical Summary}

A 20-year-old apparently healthy man presented to the emergency department with chest discomfort that had begun the previous day, when he was hit in the chest during karate. He had deep retrosternal discomfort that was relatively mild, nonradiating, and nonpleuritic. The pain intensified during the ensuing hours and led to several episodes of lightheadedness that led him to seek medical attention. On physical examination, the patient had mild kyphosis but otherwise normal habitus $(172 \mathrm{~cm} / 60 \mathrm{~kg})$; he was not diaphoretic. Heart sounds were normal and peripheral pulsations were symmetric, blood pressure was 140/80 mm Hg in both arms. Electrocardiography showed sinus rhythm with 75 beats/min without abnormalities. The patient had mild hypertelorism, but he did not have ocular or skeletal signs of Marfan syndrome, skin hyperlaxity, increased joint mobility, or bifid uvula. His medical history showed cosmetic surgery for pectus excavatum several years ago and several subluxations of the knee joints during sports. On direct questioning

From the Department of Cardiology and Cardiothoracic Surgery, Institute of Clinical and Experimental Medicine-IKEM, ${ }^{a}$ Prague, Czech Republic; Department of Radiology, Nemocnice Na Homolce, ${ }^{b}$ Prague, Czech Republic; and Center for Medical Genetics, Ghent University Hospital, ${ }^{\mathrm{c}}$ Ghent, Belgium.

The authors are supported by grant MZO-00023001 from the Czech State Department of Health.

Received for publication Oct 13, 2007; accepted for publication Jan 7, 2008.

Address for reprints: Vojtech Melenovsky, MD, PhD, Department of Cardiology, Institute for Clinical and Experimental Medicine-IKEM, Videnska 1958/9, Prague 4, 140 21, Czech Republic (E-mail: vojtech.melenovsky@ ikem.cz).

J Thorac Cardiovasc Surg 2008;135:1174-5

$0022-5223 / \$ 34.00$

Copyright (C) 2008 by The American Association for Thoracic Surgery doi:10.1016/j.jtcvs.2008.01.003 about family history, the patient mentioned that his mother and grandmother had both died suddenly of arterial dissection at age 29 years. This information led to further testing in the emergency department even though the clinical picture was not suggestive of acute aortic dissection. A chest radiogram demonstrated a widened mediastinum, and an echocardiogram showed dilatation of the proximal aorta with a luminal flap; cardiac structure and function were otherwise normal. The computed tomographic angiogram (Figure 1, A) confirmed extensive aortic root dissection, extending from the sinotubular junction to the brachiocephalic trunk, sparing the coronary sinuses and arch branches. The patient underwent an urgent Bentall procedure with replacement of the aortic valve, ascending aorta, and aortic arch (hemiarch) with a valved graft in deep hypothermia $\left(25^{\circ} \mathrm{C}\right)$ and circulatory arrest (67 minutes) with antegrade cerebral perfusion. The patient's recovery after surgery was uneventful, and he was discharged 8 days after the operation. The patient was advised to avoid vigorous physical activities and is currently treated with a beta-blocker, warfarin, and losartan.

Histologic analysis of the aortic tissue (Figure 1, B, Sirius Red stain) showed cystic medionecrosis. Postoperative magnetic resonance angiography (Figure 2, A) demonstrated the rest of the aorta to be of normal size, but diffuse arterial elongations and tortuousities were most apparent in the carotid, vertebral, and basilar territories (Figure 2, $A$ and $B$ ). Several features of this case (non-Marfan aortic aneurysm, systemic arterial tortuosity, autosomal dominant inheritance) were suggestive of the recently described Loeys-Dietz syndrome (LDS), ${ }^{1}$ causally linked to the mutations in the genes encoding transforming growth factor (TGF)- $\beta$ receptors I or II (TGFBR 1/2). The sequencing of TGFBRI/2 coding regions of the proband's DNA identified a distinctive Arg487Gln mutation in exon 9 of TGFBRl, not present in any of the genotyped unaffected relatives (Figure E1), supporting the causality of the mutation. None of the patient's screened living relatives (Figure E1, asterisks) had similar aortic involvement.

\section{Discussion}

Aortic dissections occur typically in older hypertensive patients with a peak incidence in the sixth decade, but $7 \%$ of cases occur in young subjects (aged $<40$ years), ${ }^{2}$ frequently with atypical presentation. Aortic dissection in this subgroup is most often related to an underlying connective tissue disease, such as Marfan syndrome, vascular Ehlers-Danlos syndrome, nonsyndromic familial thoracic aortic aneurysm with dissections, or the recently described LDS. LDS is characterized by generalized arterial tortuosity, craniofacial features (hypertelorism, bifid uvula), and aggressive aortic aneurysm disease. Because arterial involvement is more extensive and the propensity to rupture is higher than in other diseases affecting the aorta, early recognition of this phenotype is crucial for proper management. Genetic testing, screening of family members, and meticulous surveillance of arterial tree with a low threshold for prophylactic surgical intervention has been recently recommended on the basis of surgical experience with 71 LDS cases. ${ }^{3}$ The patients should annually undergo head-to-pelvis arterial tree imaging, and those with aortic root or 

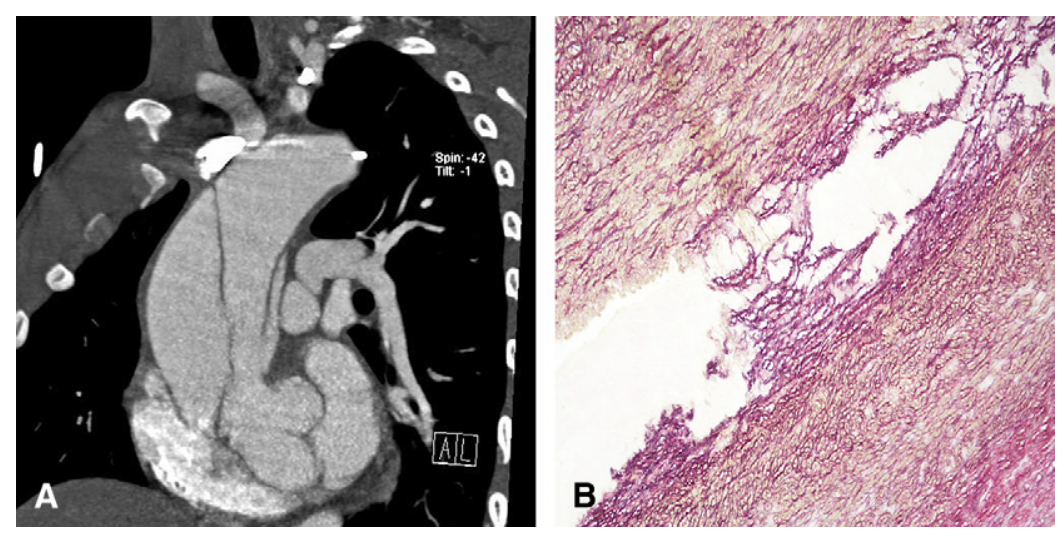

Figure 1. A, Computed tomographic angiogram at the presentation demonstrated extensive dissection of the aortic root. B, Histologic analysis of the aortic tissue (Sirius red stain) showed cystic medionecrosis.
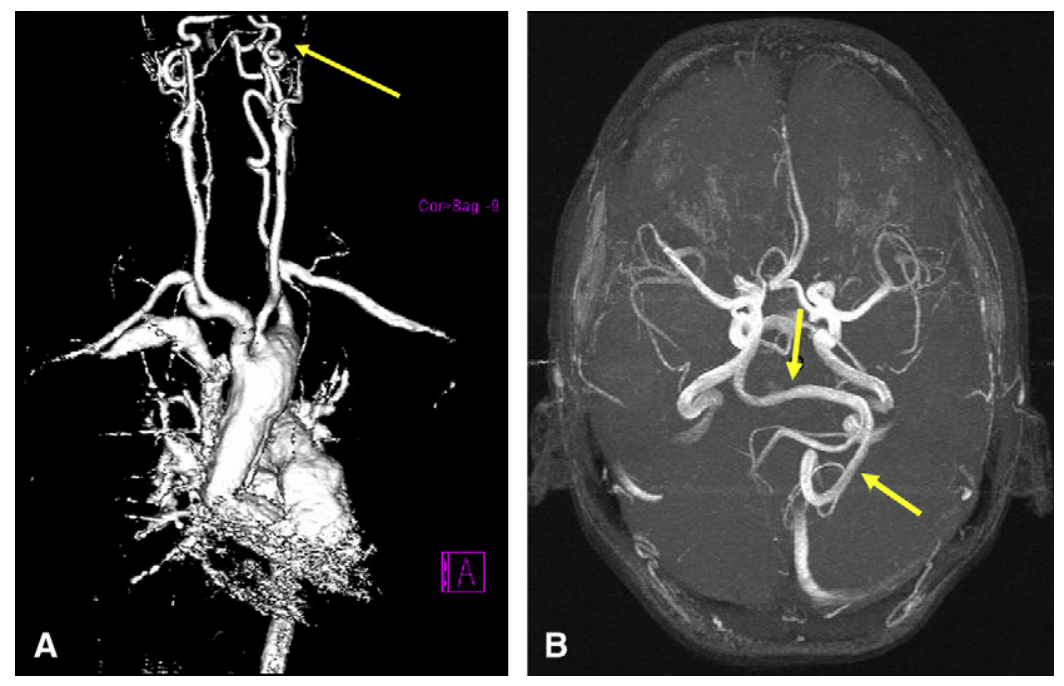

Figure 2. Postoperative magnetic resonance angiogram demonstrated diffuse arterial ellongations and tortuosities (arrows), most apparent in carotid + vertebral $(A)$ and basilar $(B)$ territories.

abdominal aorta greater than $4 \mathrm{~cm}$ or rapid expansion of greater than $0.5 \mathrm{~cm}$ in 1 year should be referred for aneurysm repair.

\section{References}

1. Loeys BL, Schwarze U, Holm T, Callewaert BL, Thomas GH, Pannu H, et al. Aneurysm syndromes caused by mutations in the TGF-beta receptor. N Engl J Med. 2006;355:788-98.
2. Januzzi JL, Isselbacher EM, Fattori R, Cooper JV, Smith DE, Fang J, et al. International Registry of Aortic Dissection (IRAD). Characterizing the young patient with aortic dissection: results from the International Registry of Aortic Dissection (IRAD). J Am Coll Cardiol. 2004;43: 665-9.

3. Williams JA, Loeys BL, Nwakanma LU, Dietz HC, Spevak PJ, Patel ND, et al. Early surgical experience with Loeys-Dietz: a new syndrome of aggressive thoracic aortic aneurysm disease. Ann Thorac Surg. 2007;83: S757-63. 


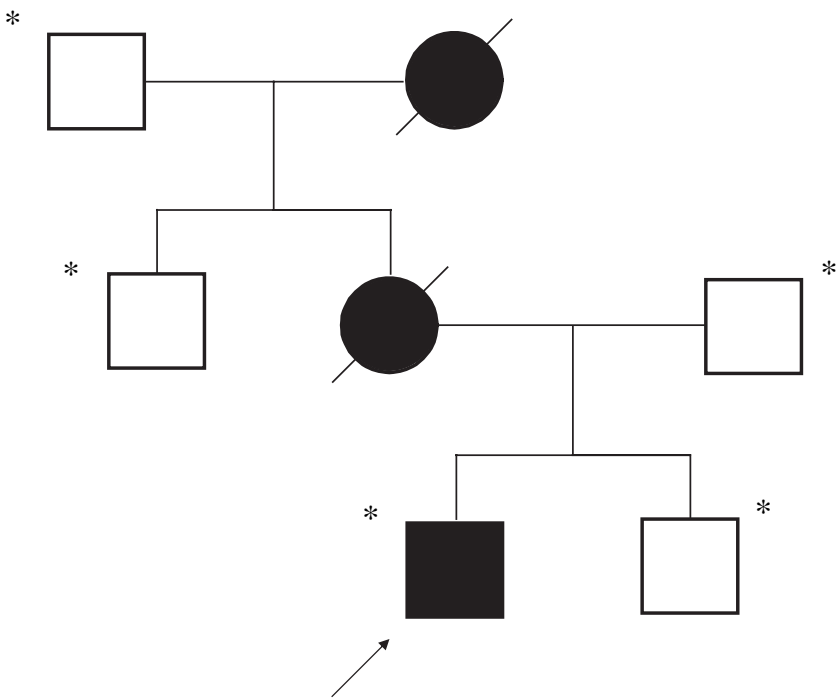

Figure E1. Family tree presenting affected (dark) and unaffected relatives of the proband (arrow). Asterisks denote genotyped individuals. 